\title{
Optic neuropathy following radiotherapy for Cushing's disease followed by the diagnosis of pituitary carcinoma
}

Krystallenia Alexandraki, Georgios Boutzios, Georgios Nikolopoulos, Panagoula Roussaki, Maria Chrysochoou, Eleftherios Chatzellis, Marina Tsoli, Maria Kaltsatou, Panayiotis Moschouris, Gregory Kaltsas

Endocrine Unit, Department of Pathophysiology, University of Athens, Medical School, Laiko Hospital, Athens, Greece

\section{BACKGROUND}

Adrenocorticotrophin secreting pituitary carcinomas are rare neoplasms but there are not factors to predict their aggressive evolution nor a standard practice to their management.

\section{CASE REPORT}

43 year old female patient presented in 1990 with Cushing's syndrome due to adrenocorticotropin secreting pituitary microadenoma

\section{THERAPEUTIC MANAGEMENT}

1990: Trans-sphenoidal surgery with cure (post-operative cortisol serum levels $<50 \mathrm{nmol} / \mathrm{L})$.

2000: clinical, biochemical and imaging recurrence (10 years after her first remission)

2001: repeat surgery with cure (post-operative cortisol serum levels $<50 \mathrm{nmol} / \mathrm{L})$

2002: recurrence

2002-2004: Ketoconazole (increased doses up to $1200 \mathrm{mg}$ ) + cabergoline + somatostatin analogues therapy

2005: no tumour was documented in imaging studies; she received external beam irradiation (in total a dose as high as $54 \mathrm{~Gy}$ ) 2005: increased doses of metyrapone up to $4000 \mathrm{mg}$ and ketoconazole up to $1200 \mathrm{mg}$

2005: UNCONTROLLED hypercortisolaemia $\rightarrow$ bilateral adrenalectomy
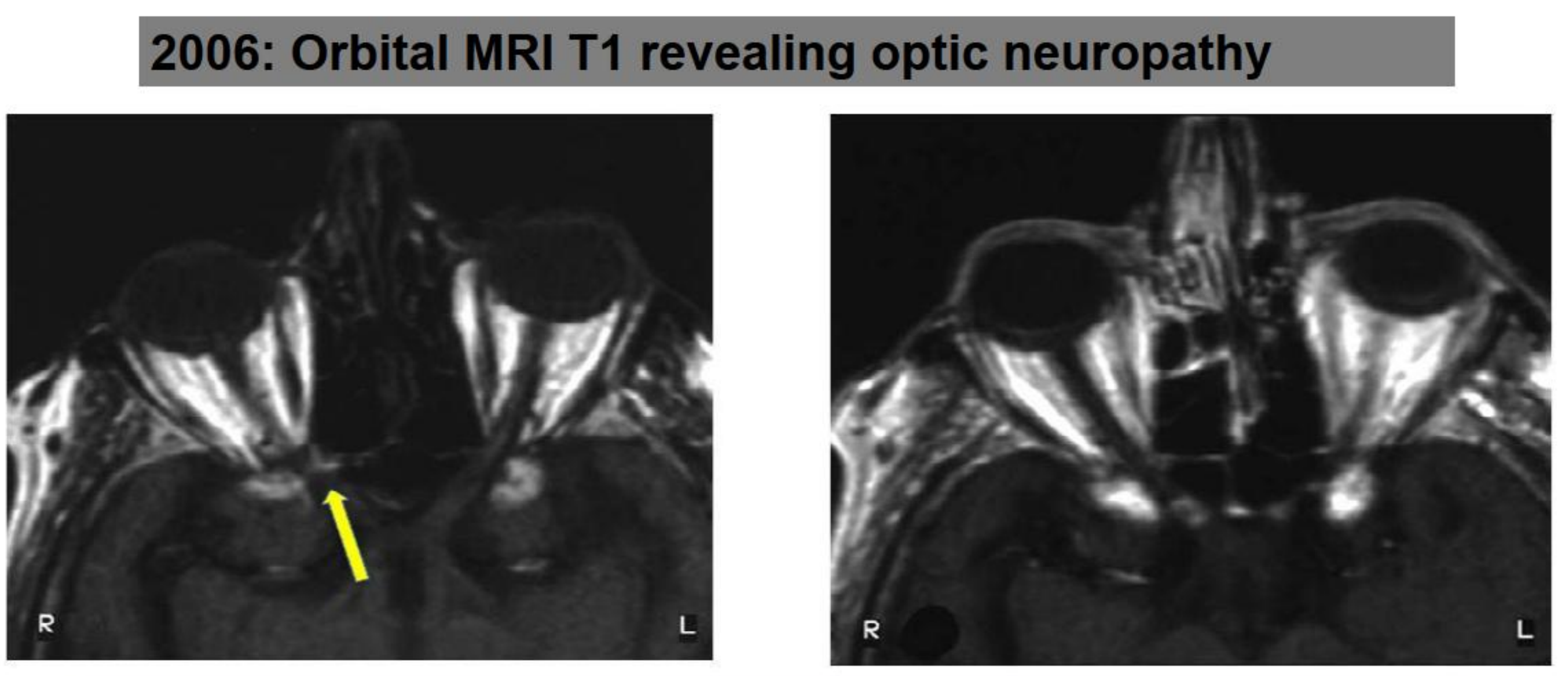

5 days IV administration of corticosteroids $\rightarrow$ aggravation

6/2006 : Hyperbaric oxygen therapy (20 cycles, 2 Atm for $30 \mathrm{~min}$ )- no result

Visual acuitv: $\mathrm{n} / 10$ in both eves

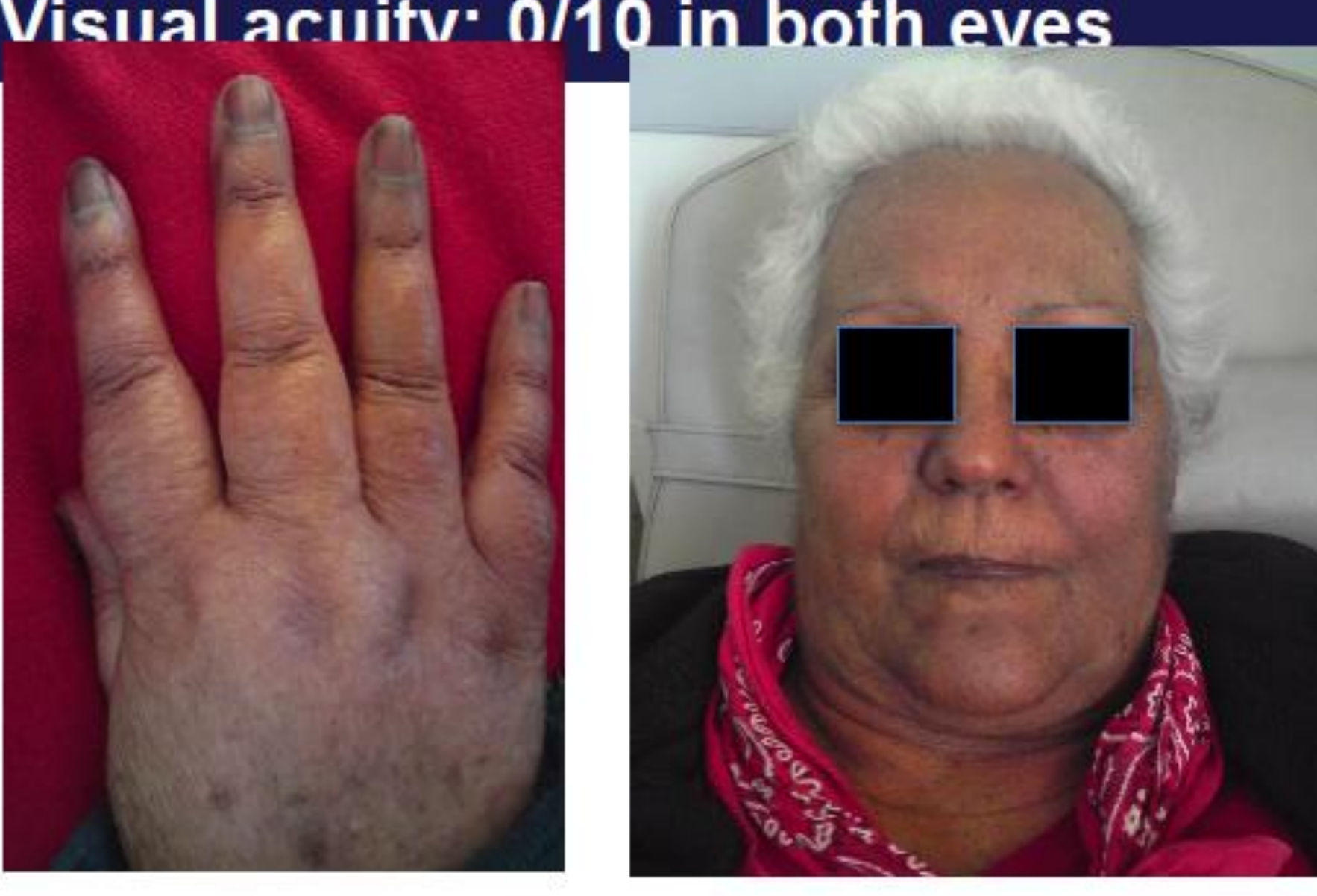

2009-2010: АCTH:

$5.858 \rightarrow 22.240 \mathrm{pg} / \mathrm{ml}$

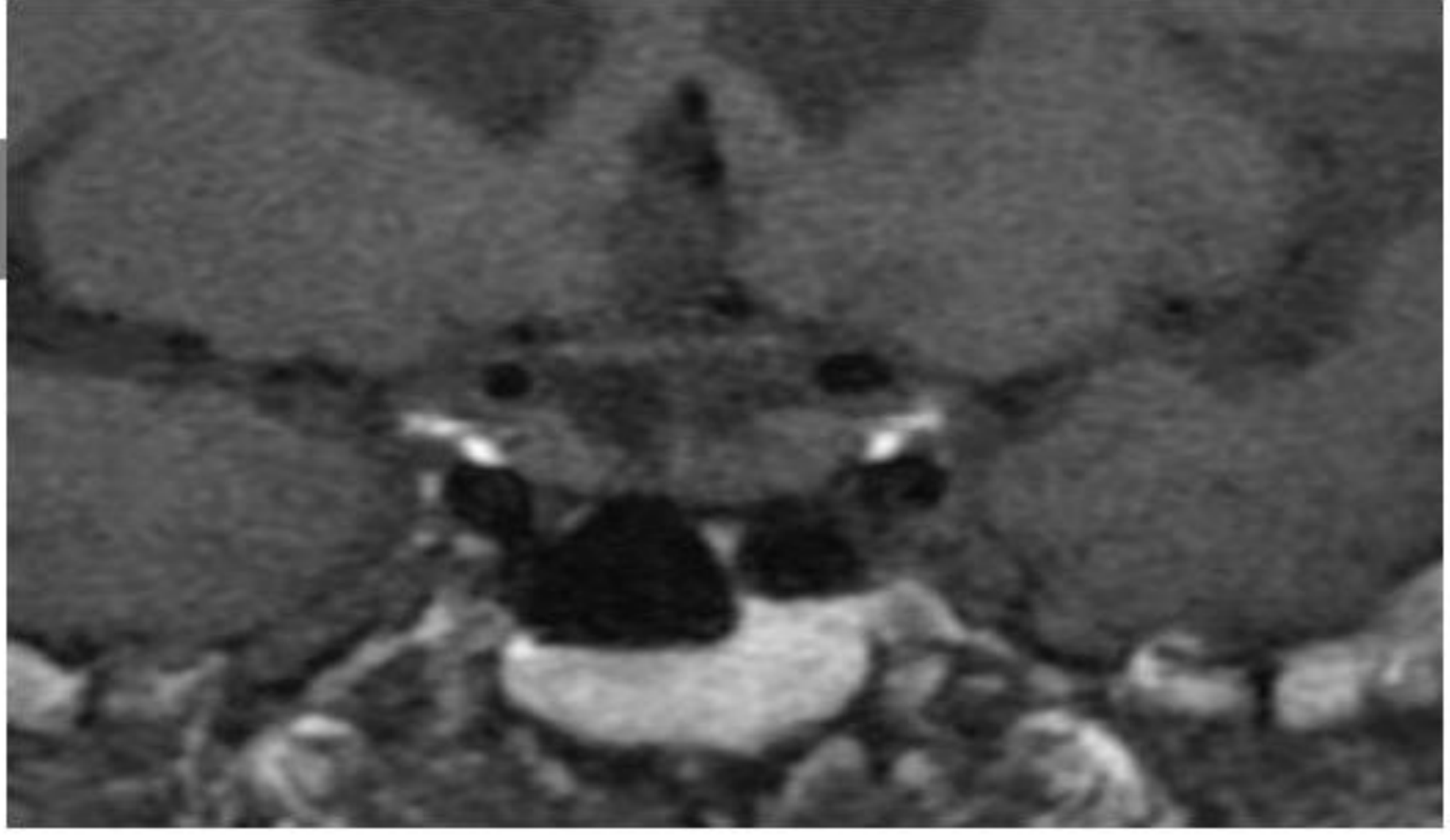

2008: ACTH: 200 pg/ml (9-52)

Pituitary MRI: post-op changes

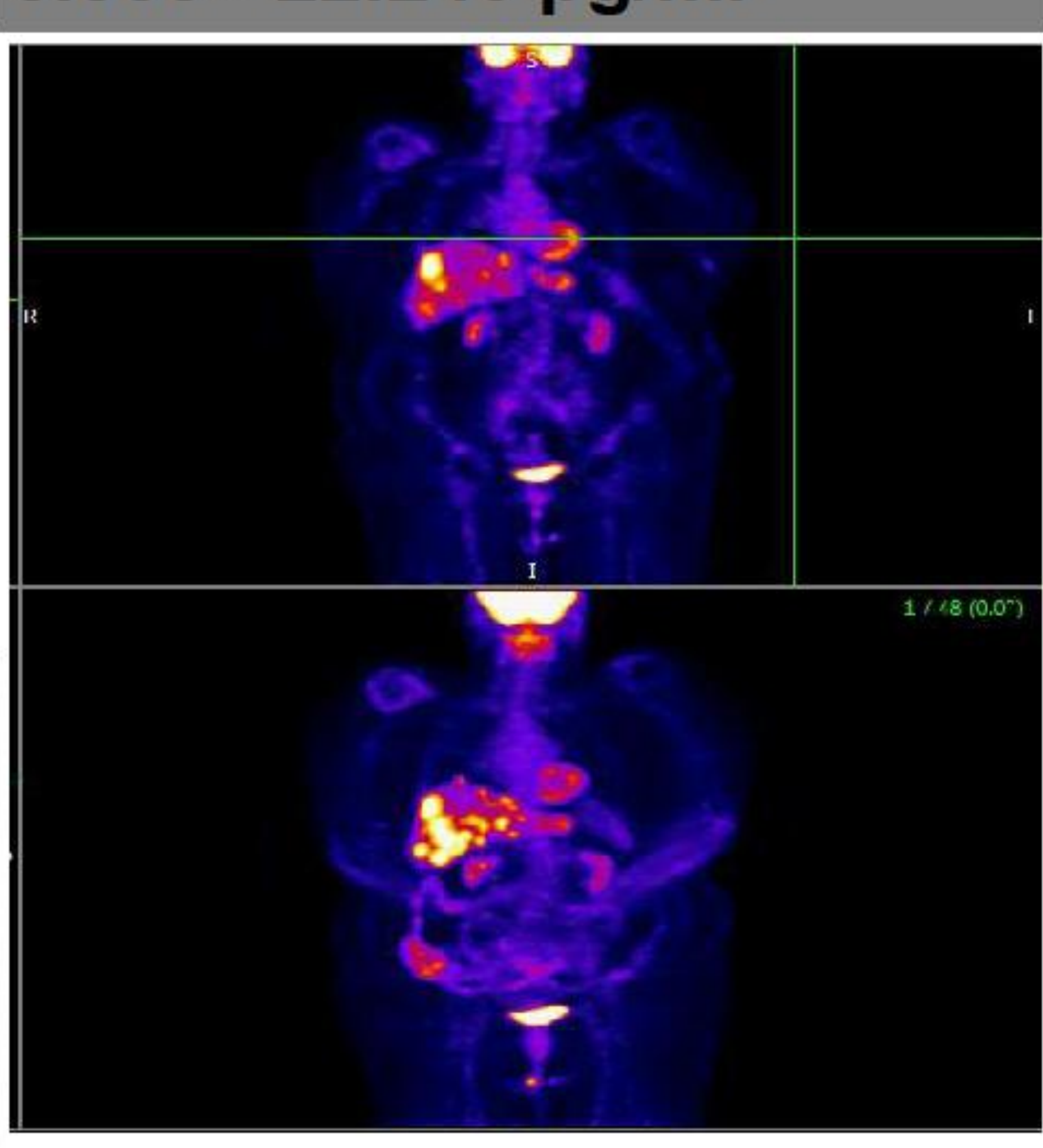

2010: FDG-PET Scan: multiple metastatic deposits in liver (SUVmax 21,2 -12), pituitary: (SUVmax 9,1); no lymph nodes/ bones/ brain

References

Koumarianou et al. Endocrine-Related Cancer ,2012; Heaney.JCEM

96:3649-3660,2011; Dudziak et al. September 2011, 96:2665-9; Ann

I. McCormack. Eur J Clin Invest 2011; 41: 1133-48

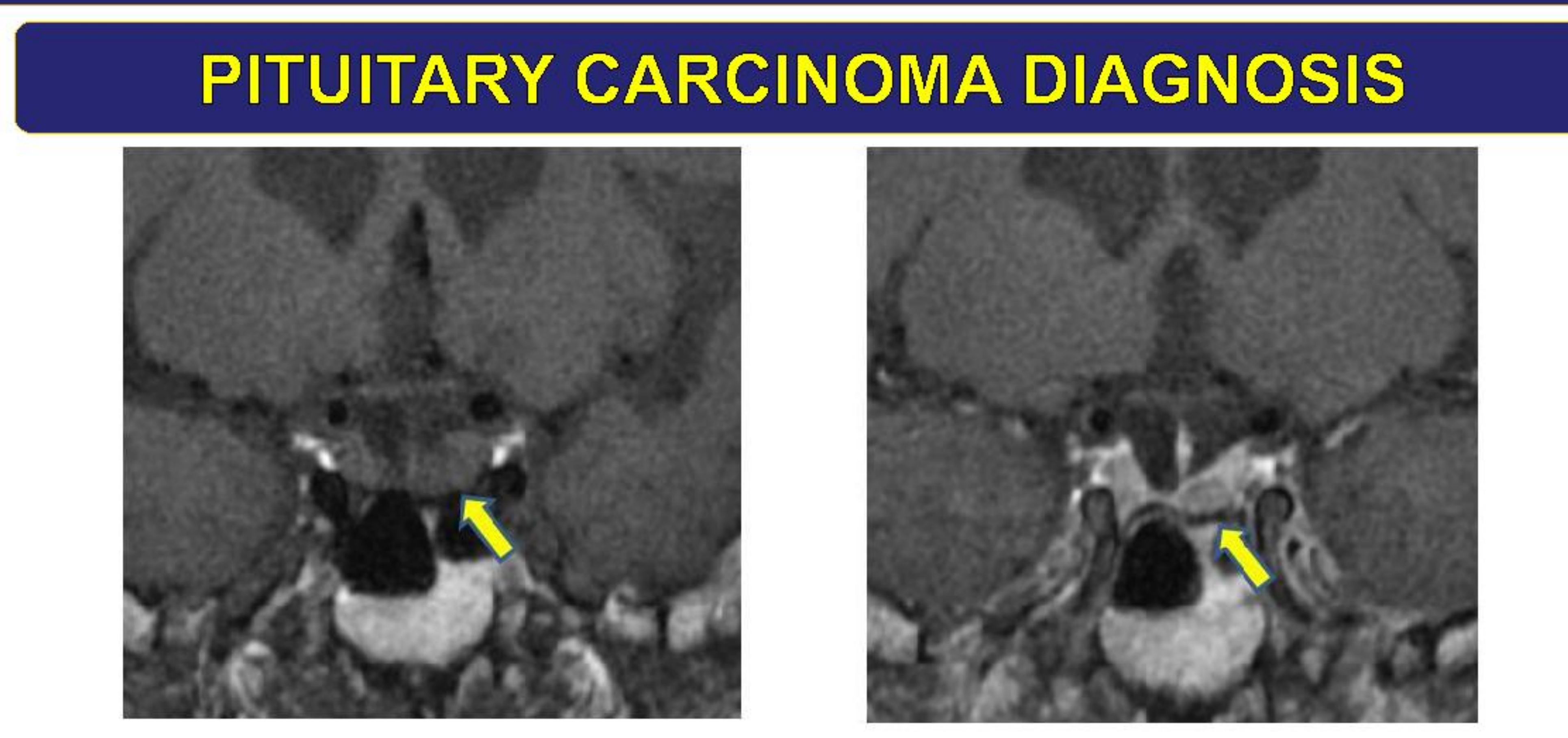

Presence of lesion $7.5 \mathrm{~mm}$ in the left lateral part of pituitary, not clear margin . Optic chiasm atrophy.

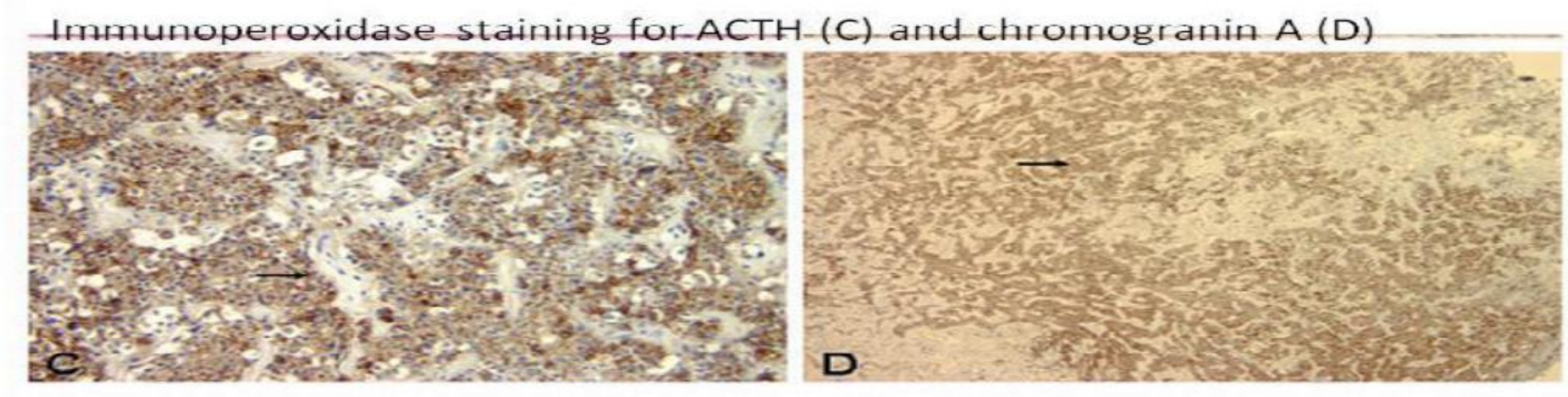

29/12/2012.Liver biopsy. Well differentiated endocrine carcinoma. Immunohistochemistry: Ker 18 (+), Syn (+), CgA (+), CD $56(+)$, Ki$67=3 \%$, ACTH (+). OCTREOSCAN : (-)ve

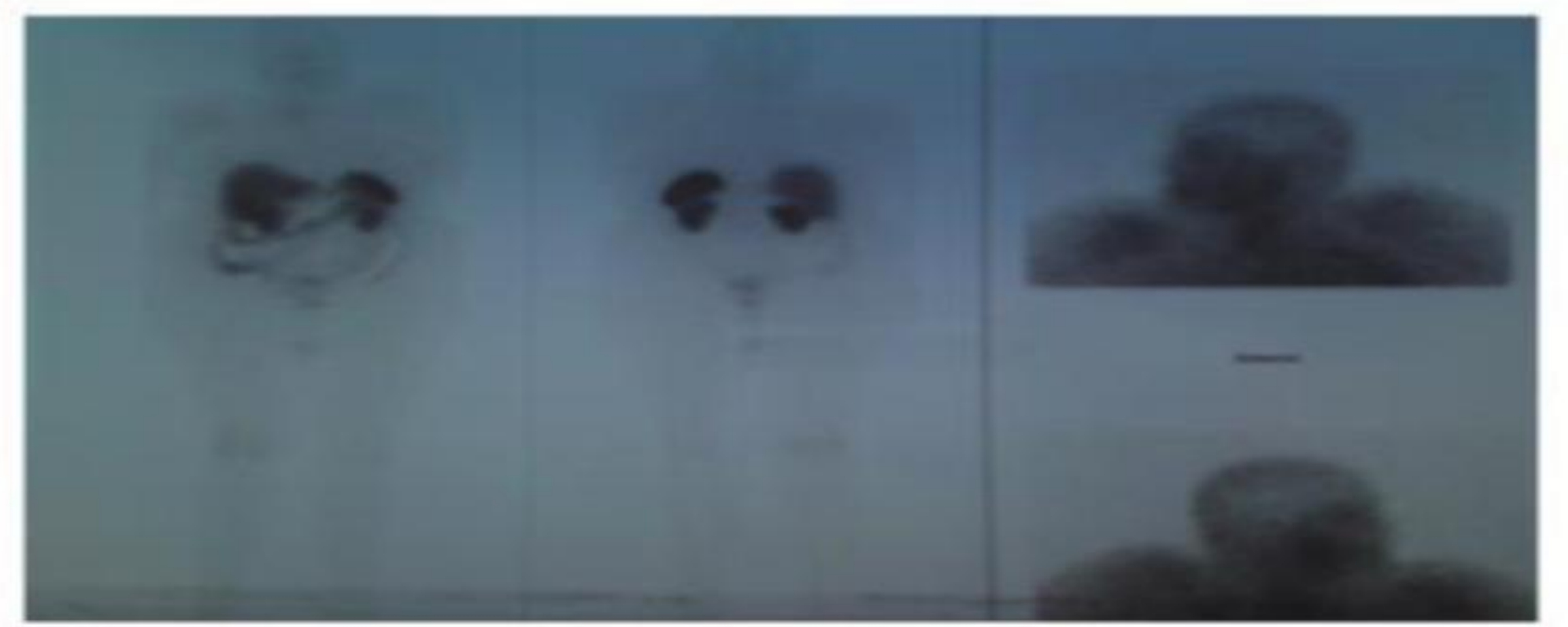

THERAPEUTIC MANAGEMENT

$\$ 3$ cycles of temozolamide (Temodal) $100 \mathrm{mg} 5$ days / week PO + bevacizumab IV (Avastin) $200 \mathrm{mg} /$ week

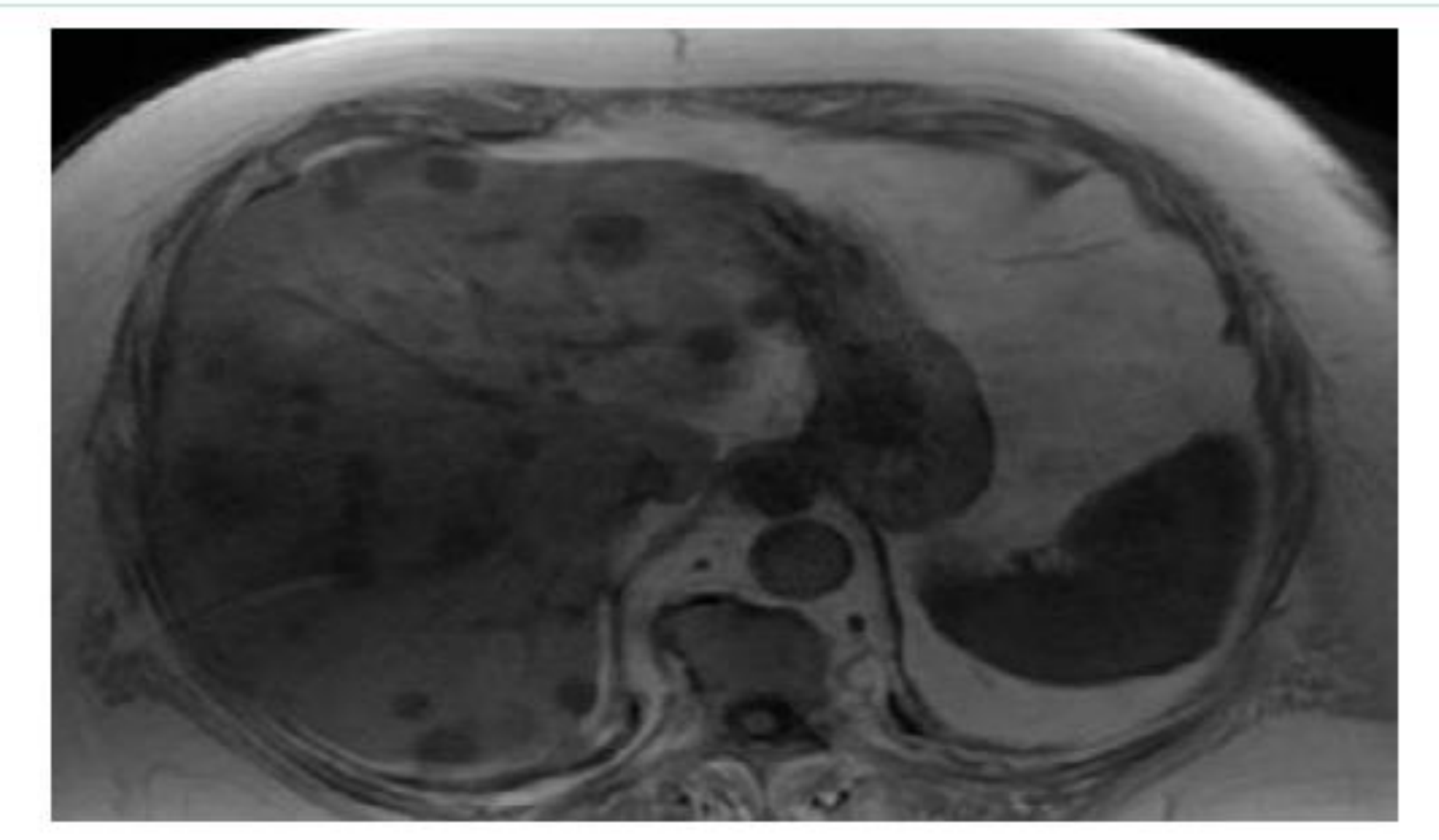

Biochemical Response

Imaging Deterioration

LOW DIFFERENTIATION

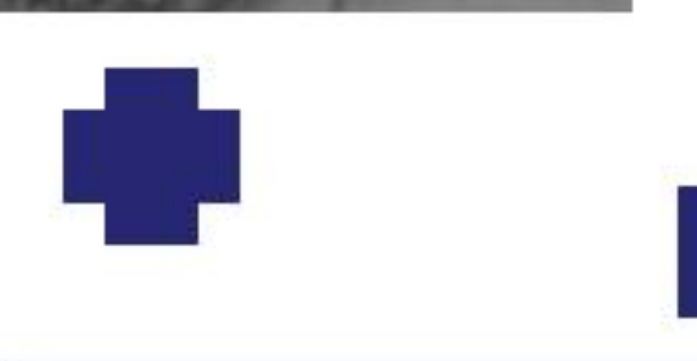

Dedifferentiation?

ACTH: $223.019 \rightarrow 89.900 \mathrm{pg} / \mathrm{m}$ BUT MRI ABDOMEN $\rightarrow \uparrow$ LESIONS SIZE; MAX 39×39mm $\rightarrow$ $52 \times 51 \mathrm{~mm}(33.3 \% \uparrow)$

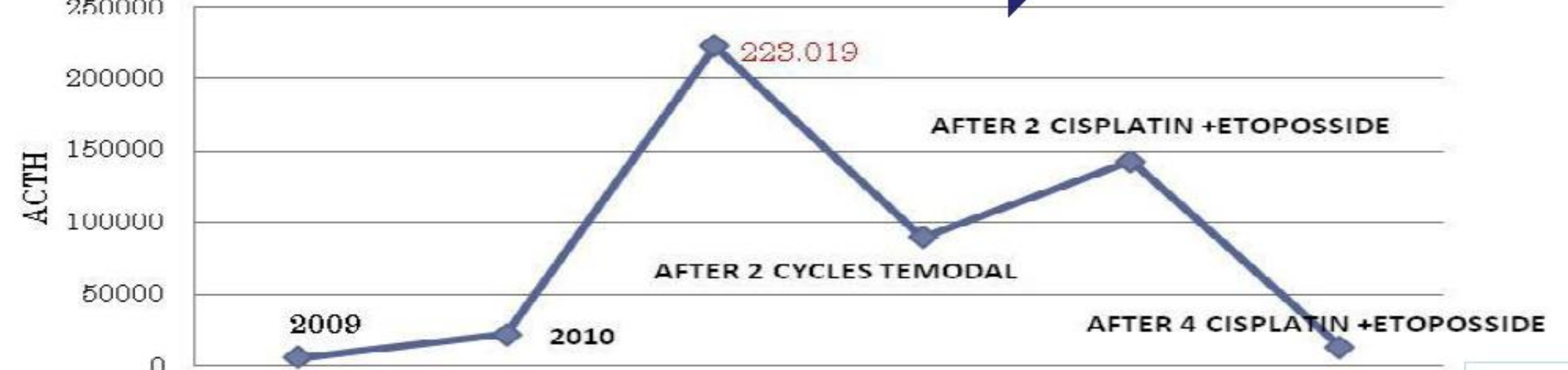

ACTH : $13.375 \rightarrow 88.590 \mathrm{pg} / \mathrm{ml}(9-52)$ MRI ABDOMEN $\rightarrow \uparrow$ LESIONS SIZE+NUMBER$$
\text { Dedi }
$$

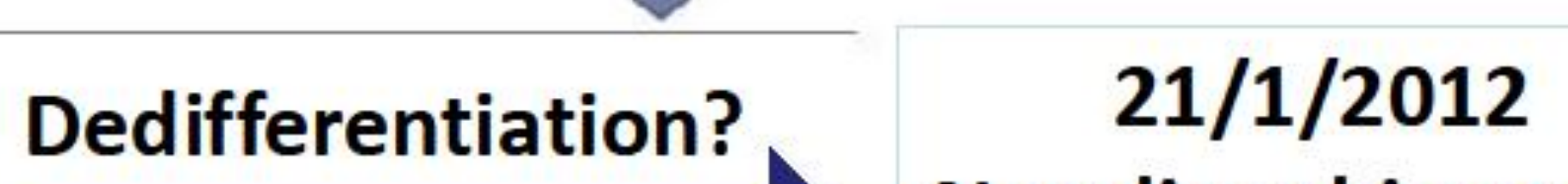

$21 / 1 / 2012$ Ki67 $\sim 8 \%$

MGMT (methylguanine DNA methyltransferase) Methylation specific PCR (MS11/9/2012 RADIOFREQUENCY RIGHT LIVER LOBE

P): LOW BUT Temozolamide + Bevacizumab

SEPSIS $\rightarrow$ MYELOTOXICITY $\rightarrow$ CONSERVATIVE TREATMENT

DECEASED : 19/9/2014

\section{CONCLUSIONS}

The appearance of pituitary carcinoma cannot be excluded by the cure post trans-sphenoidal surgery nor be prevented by a high dose of irradiation even this is so high to cause radiation-induced optic neuropathy 